\title{
Corrective surgery for a patient with a giant right pulmonary artery developing 40 years after a Waterston-Cooley shunt for pulmonary stenosis and ventricular septal defect
} \author{
Seoul and Bucheon, Republic of Korea
}

Jae Gun Kwak, MD, PhD, ${ }^{a}$ Chang-Ha Lee, MD, PhD, ${ }^{b}$ Eun Seok Choi, MD, ${ }^{b}$ and Seong Ho Kim, MD, PhD,

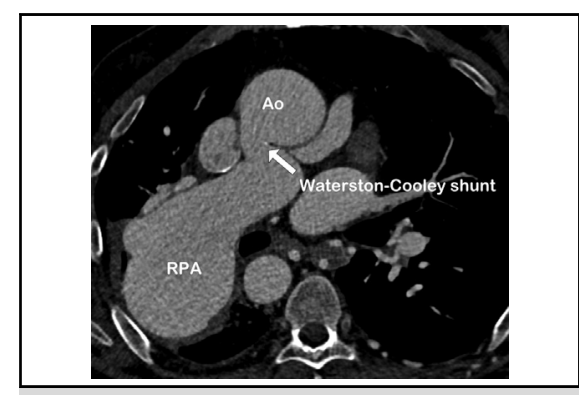

Severely enlarged right pulmonary artery and Waterston-Cooley anastomosis site.

\section{Central Message}

We performed a corrective surgery for a patient with pulmonary stenosis and ventricular septal defect who had been in 50-year palliative status with Waterston-Cooley and Blalock-Taussig shunts.

See Editorial Commentary page e37. the age of 40 years because of brain abscess, after 20-years without follow-up the after Waterston-Cooley shunt operation, we performed a cardiac catheterization. Catheterization showed an almost occluded LMBT shunt, a small left pulmonary artery (LPA), and a severely dilated right pulmonary artery (RPA). The RPA exhibited hypertensive changes (systolic and mean pressures of 72 and $56 \mathrm{~mm} \mathrm{Hg}$, respectively), attributable to the Waterston-Cooley shunt. At this time, we constructed a new 7-mm-diameter LMBT shunt to rehabilitate the small LPA and banded around the anastomosis site of the Waterston-Cooley shunt to halt progressive dilatation and hypertensive change in the RPA. The patient was unavailable for follow-up until the age of 48 years, at which time his heart function was reevaluated. Cardiac catheterization study revealed aggravated RPA pressure (systolic and mean pressures of 91 and $58 \mathrm{~mm} \mathrm{Hg}$, respectively), despite the previous Waterston-Cooley shunt banding. Computed tomography confirmed a welldeveloped LPA (14 mm in diameter), a patent LMBT shunt, and a dilated RPA (maximum diameter of $42 \mathrm{~mm}$ in the lung parenchyma). The patient visited the emergency department with hematemesis at the age of 56 years, after another 8-year unavailability for follow-up. The LMBT shunt was still patent, but the RPA remained enlarged (69 $\mathrm{mm}$ in diameter) on computed tomography (Figure 1, $A$ through $D$ ). Fortunately, a catheterization study

showed a preserved LPA vascular bed. Atrial fibrillation was evident. His functional class was between 2 and 3 . We explained to the patient the possibility of sudden rupture of his severely dilated RPA and the necessity of eliminating the high aortic pressure on the RPA to reduce this risk. Because the LPA was well preserved, we considered it possible to perform corrective surgery rather than palliative operation. The patient agreed to surgical treatment.

We divided the LMBT and Waterston-Cooley shunts, closed the ventricular septal defect, performed RPA reduction plasty to the hilar level with resection of the anterior part of the dilated RPA wall and anastomosis of the resected margin, and resected hypertrophied right ventricular muscle. The pulmonary valve was not markedly dysplastic, so we saved the patient's native valve with a simple commissurotomy. We created an $8-\mathrm{mm}$ diameter atrial septal defect because of high RPA pressure and performed a maze procedure. Postoperatively, the patient's New York Heart Association functional class was much improved to between 1 and 2, and RPA vascularity was significantly reduced on chest radiography (Figure 2). Right ventricular function improved and pulmonary valve function was acceptable, with $45 \mathrm{~mm} \mathrm{Hg}$ of pressure gradient 


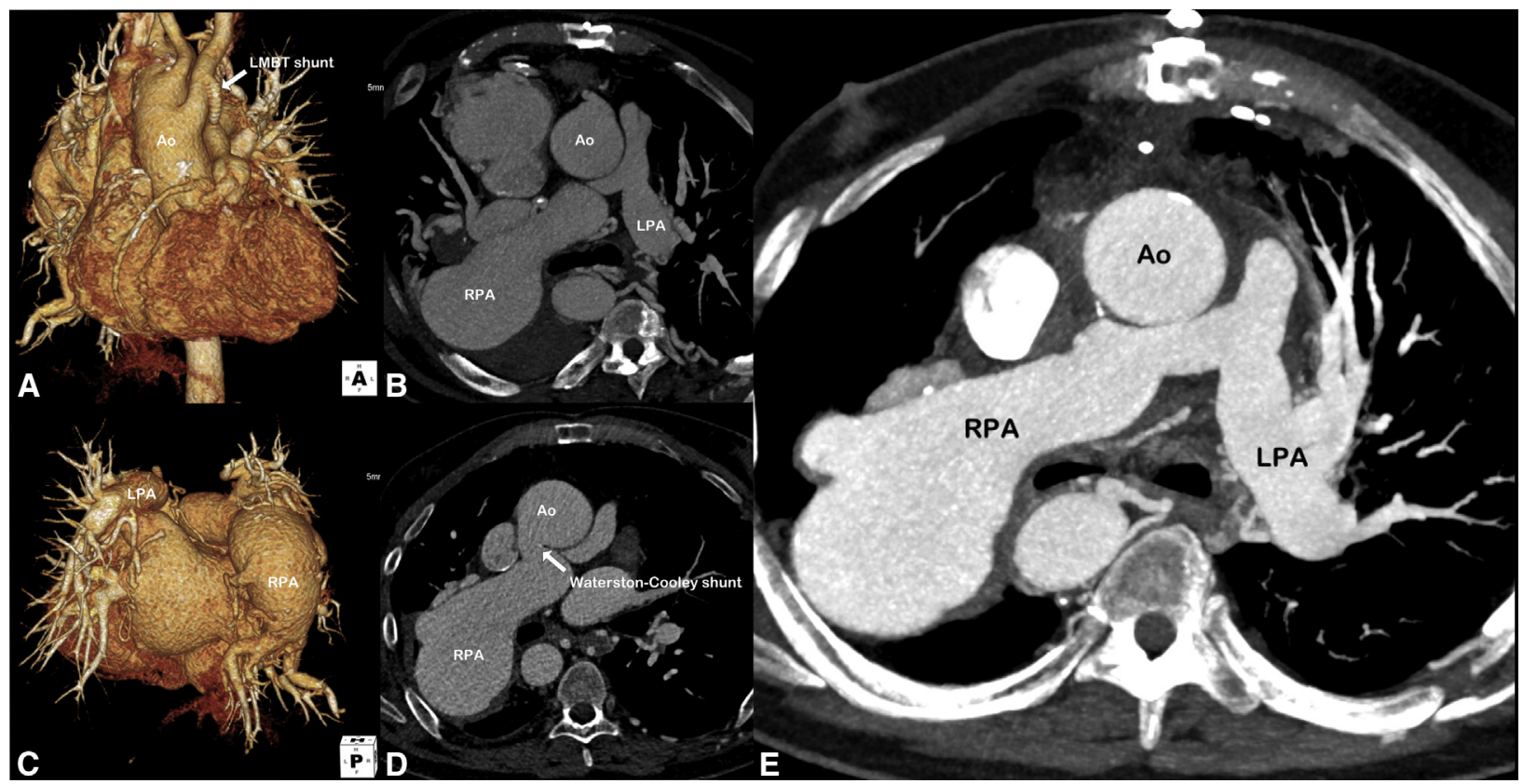

FIGURE 1. Preoperative (A-D) and postoperative (E) computed tomographic images. A, In an anterior view of the heart on 3-dimensional computed tomography, the white arrow indicates a left-sided modified Blalock-Taussig ( $L M B T$ ) shunt. B, A computed tomographic axial image shows a well-grown left pulmonary artery $(L P A)$ after left-sided modified Blalock-Taussig shunt and an enlarged right pulmonary artery (RPA), especially in lung parenchyma. C, A posterior view of the heart on 3-dimensional reconstructed computed tomography clearly shows dilated right pulmonary artery. $\mathrm{D}$, A computed tomographic axial image shows a Waterston-Cooley anastomosis site between the ascending aorta (Ao) and the right pulmonary artery. E, A computed tomographic axial image shows well-arranged branch pulmonary arteries after corrective surgery.

and mild regurgitation on echocardiography. The direction of shunt flow through the atrial septal defect was left to right.

\section{DISCUSSION}

Since Blalock and Taussig treated a patient with tetralogy of Fallot with a Blalock-Taussig shunt, certain modifications of systemic-to-pulmonary arterial shunt have been developed to increase pulmonary blood flow. ${ }^{1-3}$ Other than the modified Blalock-Taussig shunt, other forms of systemic-to-pulmonary shunts have become increasingly unpopular because of their various disadvantages. Among them, the Waterston-Cooley shunt has been abandoned because it is associated with excessive pulmonary blood flow, triggering congestive heart failure, pulmonary

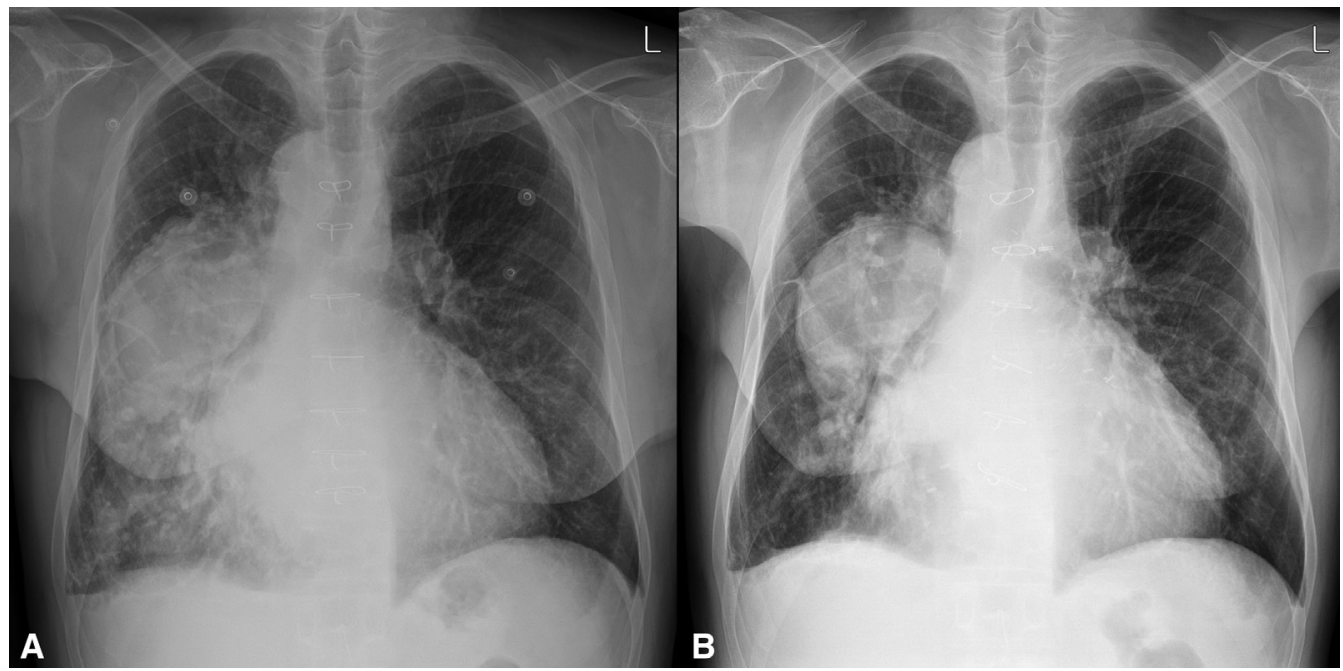

FIGURE 2. Posteroanterior chest radiographic images. A, Preoperative image. B, Postoperative image 1 month after corrective surgery. 
hypertension, RPA aneurysmal change or distortion, or obstruction of the RPA-aorta anastomosis site with time. ${ }^{4,5}$ We still sometimes encounter patients who underwent such old-fashioned shunt procedures as palliative treatments, however, and, although even more rarely, we sometimes see those who retain palliative status for many years.

In this case, the patient had undergone a WaterstonCooley shunt procedure in the early era of cardiac surgery and unfortunately had not had sufficient follow-up. As a result, his RPA became severely dilated and exhibited hypertensive changes with time. Belated banding at the Waterston-Cooley shunt site could not halt or restore pulmonary vascular changes in the right lung, and in addition to this the severely dilated RPA appeared to be at great risk for rupture. Even though we could not approach the maximally dilated area because it was located in lung parenchyma, we considered it possible that interruption of systemic pressure on the RPA and reduction of RPA size would reduce the likelihood of sudden rupture. We were concerned that the RPA would not accept pulmonary blood flow well after corrective surgery because of hypertensive change, but we also expected that the preserved LPA vascular bed would be acceptable for anatomically corrected status if we made an atrial septal defect. The patient's postoperative course was satisfactory, but strict regular follow-up will be required to evaluate change of pressure and size of the dilated RPA (Figure 1,E) as well as to check the patient's symptoms and signs, such as hemoptysis or respiratory difficulty. Right pneumonectomy could be an option in a critical situation.

\section{References}

1. Potts WJ, Smith S, Gibson S. Anastomosis of the aorta to a pulmonary artery; certain types in congenital heart disease. J Am Med Assoc. 1946;132: 627-31.

2. Waterston DJ. [Treatment of Fallot's tetralogy in children under 1 year of age] Rozhl Chir. 1962;41:181-3. Article in Czech.

3. Cooley DA, Hallman GL. Intrapericardial aortic-right pulmonary arterial anastomosis. Surg Gynecol Obstet. 1966;122:1084-6.

4. Rao PS, Ellison RG. The cause of kinking of the right pulmonary artery in the Waterston anastomosis. A growth phenomenon. J Thorac Cardiovasc Surg. 1978;76:126-9.

5. Reitman MJ, Galioto FM Jr, el-Said GM, Cooley DA, Hallman GL, McNamara DG. Ascending aorta to right pulmonary artery anastomosis. Immediate results in 123 patients and one month to six year follow-up in 74 patients. Circulation. 1974;49:952-7. 